\title{
Puerarin protects against human retinal endothelial cells injury induced by high glucose via regulating miR-16-5p/CASP1 axis
}

\author{
Jie Zhang ${ }^{1,2}$, Yueqin $\mathrm{Chen}^{3}$ and Weiping $\mathrm{Gao}^{1}$ \\ ${ }^{1}$ Department of Ophthalmology, The Affiliated Hospital to Nanjing University of Chinese Medicine, Nanjing, Jiangsu, \\ China \\ ${ }^{2}$ Department of Ophthalmology, Yizheng Hospital of Chinese Medicine, Yizheng, Jiangsu, China \\ ${ }^{3}$ Department of Ophthalmology, Jinling Hospital Nanjing, Jiangsu, China
}

\begin{abstract}
We aimed to investigate the effects of puerarin on high glucose (HG)-induced human retinal endothelial cells (HRECs) injury. Cells were exposed to puerarin in the presence or absence of HG challenge. Cell viability was determined using a CCK- 8 assay. Then, the levels of the oxidative stress-related factors were evaluated using corresponding kits. Pyroptosis was assessed by measurement of gasdermin-N domain (GSDMD-N) and caspase-1 (CASP1) expression. Subsequently, miR-16-5p expression was detected using RT-qPCR. The levels of oxidative stress and pyroptosis were examined after miR-16-5p silencing. The Starbase database predicted that CASP1 is a potential target of miR-16-5p, which was verified through a luciferase reporter assay. Moreover, CASP1 expression was determined after miR-16-5p silencing in HG-stimulated HRECs with puerarin exposure. Results revealed that puerarin significantly enhanced cell viability and inhibited oxidative stress. Furthermore, puerarin markedly downregulated GSDMD-N and CASP1 expression, and miR-16-5p level was notably inhibited in HG-stimulated HRECs, which was reversed after puerarin intervention. Following transfection with miR-16-5p inhibitor, the effects of puerarin on cell viability, oxidative stress and pyroptosis were attenuated in HG-induced HRECs. CASP1 was confirmed as a direct target gene of miR-16-5p. Taken together, puerarin alleviates oxidative stress and pyroptosis in HG-stimulated HRECs through regulating the miR16-5p/CASP1 axis.
\end{abstract}

Key words: Diabetic retinopathy — Puerarin — Oxidative stress - Pyroptosis - CASP1

\section{Introduction}

Diabetic retinopathy (DR), one of the most common and prevalent microvascular complications of diabetes, is considered as the main cause of diabetes-related blindness throughout the world, and as a serious threat to human health (Liu et al. 2019). At present, although many therapeutic remedies focusing on DR have been used, there still exists a lack of effective treatments for this disease (Abu El-Asrar et al. 2013). Therefore, it is of the utmost importance to understand the underlying mechanisms and identify the therapeutic agents associated with DR.

Correspondence to: Weiping Gao, Nanjing University of Chinese Medicine, 282 Hanzhong Road, Nanjing, Jiangsu, 210029, China E-mail: gaoweiping68@126.com
Emerging evidence showed that human retinal endothelial cells (HRECs) dysfunction is the initial event of microvascular disorder in the development of DR (Zhang et al. 2018). A growing body of literature has demonstrated that oxidative stress in the retina induced by high glucose (HG) plays a pivotal role in the development and acceleration of DR (Mohamed et al. 2017; Yang et al. 2020). Pyroptosis, an emerging type of cell death, is initiated by the activation of proinflammatory caspases, including caspase-1 (CASP1) (Bergsbaken et al. 2009). A recent study has found that pyroptosis is involved in the progression of DR, which can be mediated by oxidative stress (Xi et al. 2020). Pueraria lobata (Gegen in China) root has long been used as a traditional medicine. Puerarin, the primary active ingredient of the root of Pueraria lobata, has been reported to possess various important pharmacological 
properties, such as anti-oxidant, anti-inflammatory and anti-diabetic activities (Jeon et al. 2020; Wang et al. 2020). It has been reported that puerarin protects against DR in rats (Mohamed et al. 2017). Importantly, it reduces the oxidative stress damage of the retina in DR rats by inhibiting STAT3 expression (Cai et al. 2017). Moreover, puerarin was reported to inhibit CASP1 activation by reducing reactive oxygen species (ROS) generation (Lian et al. 2019). However, the effects of puerarin on HG-induced HRECs injury remain to be elucidated.

An existing study has shown that microRNA-16 (miR-16) expression markedly decreases in HRECs cultured with HG, and miR-16 suppresses insulin resistance by reducing tumor necrosis factor alpha level, thereby it protects HRECs from hyperglycemic-induced apoptosis (Ye and Steinle 2015). Compelling evidence indicate that miR-16 attenuates retinal leukostasis through decreased pro-inflammatory signaling in HRECs (Ye et al. 2016). Moreover, puerarin was reported to upregulate miR-16 expression in bladder cancer cells (Liu et al. 2018). miRNAs belong to small non-coding RNAs of 19-24 nucleotides in length which exert crucial regulatory effects in gene expression (Ramanathan and Padmanabhan 2020). The database of Starbase predicts that CASP1 is a potential target of miR-16-5p, which plays a significant role in the regulation of pyroptosis. Therefore, it attracted our attention whether puerarin alleviates HG-induced oxidative stress and pyroptosis of HRECs by regulating miR-16-5p/ CASP1 axis.

In the present study, we sought to examine the potential protective effects of puerarin on HG-induced HRECs damage and to investigate the relationship between its effect and the modulation of miR-16-5p/CASP1 axis. Our findings may identify a novel pharmaceutical approach to the treatment of DR.

\section{Materials and Methods}

\section{Cell culture and treatment}

HRECs were purchased from the Cell Bank of Chinese Academy of Sciences and maintained in Endothelial Cell Medium containing $10 \%$ fetal bovine serum at $37^{\circ} \mathrm{C}$ in a humidified $5 \% \mathrm{CO}_{2} / 95 \%$ air atmosphere. Cells were maintained in medium supplemented with normal glucose (NG group; $5.5 \mathrm{mM}$ glucose), $5.5 \mathrm{mM}$ glucose $+22.5 \mathrm{mM}$ mannitol (MA group) and with $25 \mathrm{mM}$ glucose (HG group) for $48 \mathrm{~h}$. In the puerarin-treated group (HG+Pueararin group), HRECs were pretreated with a series of different concentrations of puerarin (purity $\geq 98 \%$; cat. no. 824355MG, Sigma-Aldrich, St. Louis, MO, USA) 10, 20 and 50 $\mu \mathrm{M}$, dissolved in dimethyl sulfoxide for $3 \mathrm{~h}$ before exposure to HG condition.
Cell viability assay

Cell viability was determined using a Cell Counting Kit-8 (CCK-8) kit purchased from Shanghai Yi Sheng Biotechnology Co., Ltd (Shanghai, China). Briefly, HRECs $\left(5 \times 10^{3} /\right.$ well $)$ were seeded in a 96 -well plate and maintained at $37^{\circ} \mathrm{C}$. After being stimulated with different concentrations of puerarin in an normal glucose or HG condition, $10-\mu$ l CCK- 8 solution was added to each well, and the plates were incubated at $37^{\circ} \mathrm{C}$ for another $2 \mathrm{~h}$ according to standard protocol. The OD values at $450 \mathrm{~nm}$ were used to reflect the cell viability of HRECs.

\section{Evaluation of oxidative stress}

In order to evaluate the levels of oxidative stress, oxidative stress biomarkers including the concentrations of ROS, malondialdehyde (MDA) as well as the activities of superoxide dismutase (SOD) and catalase (CAT) were respectively measured using ROS assay kit (cat. no. E004-1-1), MDA assay kit (cat. no. A003-4-1), SOD assay kit (cat. no. A001-1-2) and CAT assay kit (cat. no. A007-1-1) in accordance with the specification provided by the supplier. Above kits were obtained from Nanjing Jiancheng Bioengineering Institute (Nanjing, China).

\section{Immunofluorescence assay}

HRECs were plated on coverslips in 24-well plates and cultured until it reached $80 \%$ confluence. Subsequently, the cells were fixed in 4\% paraformaldehyde and permeabilized with $0.05 \%$ Triton X-100. After blockage with 5\% normal goat serum, cells were incubated with a primary antibody against gasdermin-N domain (GSDMD-N; 1:200; cat. no. bs-14287R; Bioss, Beijing, China) overnight at $4^{\circ} \mathrm{C}$, then they were washed with PBS three times, incubated with DyLight ${ }^{\mathrm{mm}}$ 488-conjugated secondary antibody (Thermo Fisher Scientific, Inc.) and stained with 4,6-diamidino-2-phenylindole (DAPI; Sigma-Aldrich; Merck KGaA). After that, the stained cells were subsequently imaged under a fluorescence microscope (Olympus Corporation).

\section{Cell transfection}

Prior to transfection, $1 \times 10^{6}$ of HRECs were seeded into a 6 -well plate. The cell monolayer was $\sim 80 \%$ confluent at the time of transfection. miR-16-5p inhibitor, its negative control (inhibitor NC), miR-16-5p mimic and the mimic NC were provided by GenePharma (Shanghai, China). Transfection experiments were conducted using Lipofectamine ${ }^{\circledast} 2000$ (Invitrogen; Thermo Fisher Scientific, Inc.) according to the standard protocol. Cells were collected $48 \mathrm{~h}$ after transfection, the success of which was evaluated using reverse 
transcription-quantitative polymerase chain reaction (RTqPCR) analysis.

\section{$R T-q P C R$ analysis}

Total RNA was extracted from cells using Trizol reagent (Invitrogen). Subsequently, cDNA was synthesized using a reverse transcription kit (Beijing Transgen Biotech Co. Ltd., China) in accordance with the specification provided by the supplier. After that, qPCR was conducted with SYBR Green Supermix (Bio-Rad Laboratories, Inc.) using the ABI 7500 system (Applied Biosystems; Thermo Fisher Scientific, Inc.). Primer sequences (Sangon Biotech, China) used in this study were as follows: miR-16-5p forward, 5'-TAGCAGCACGTAAATATTGGCG-3' and reverse, 5'-TGCGTGTCGTGGAGTC-3'; U6 forward, 5'-CTCGCTTCGGCAGCACA-3' and reverse, 5'-AACGCTTCACGAATTTGCGT-3'; CASP1 forward, 5'-TAGGATTGGTCCCCAACAAGA-3' and reverse, 5'-GAGAAACCCTTTGTCGGGTGG-3'; GAPDH forward, 5'-GGAGCGAGATCCCTCCAAAAT-3' and reverse, 5'-GGCTGTTGTCATACTTCTCATGG-3'. U6 or GAPDH expression was used as the internal control. The relative fold change of gene expression was calculated using the $2^{-\Delta \Delta C t}$ method (Livak and Schmittgen 2001).

\section{Western blotting}

Total proteins samples were extracted from the treated HRECs using RIPA lysis buffer (Beyotime, Shanghai, China). A BCA Kit (Beyotime, China) was employed for determining the concentrations of proteins. The harvestable proteins $(40 \mu \mathrm{g})$ were separated in $10 \%$ SDS-PAGE gels, followed by being transferred to polyvinylidene difluoride membranes (Millipore, Bedford, MA). Next, the unspecific bands were blocked with 5\% fat-free milk and probed with the primary antibodies overnight at $4^{\circ} \mathrm{C}$. After washing the membranes with TBST, these blots were incubated with horseradish peroxidase-conjugated secondary antibody (1:5000; cat. no. ab7090; Abcam, Cambridge, UK). Signals were developed using an Odyssey Infrared Imaging Scanner (LI-COR Biosciences) and quantified by ImageJ software (National Institutes of Health, Bethesda, MA, USA). GAPDH served as the loading control. Anti-CASP1 (1:1000; cat. no. 4199T) and anti-GAPDH (1:1000; cat. no. $5174 \mathrm{~T}$ ) antibodies were obtained from Cell Signaling Technology (Boston, MA, USA). Anti-GSDMD-N (1:1000; cat. no. ab215203) antibody was purchased from Abcam Company (Cambridge, UK).

\section{Luciferase reporter assay}

Wild-type (WT) and mutant (MUT) reporter plasmids of CASP1 that contained miR-16-5p mimic or mimic NC binding sites were synthesized by Shanghai GenePharma Co., Ltd. Cells were co-transfected with the constructed WT or MUT luciferase reporter vector using Lipofectamine 2000 (Invitrogen; Thermo Fisher Scientific, Inc.). The luciferase activity was detected by a Dual Luciferase Reporter System (Promega, Madison, WI, USA). Renilla luciferase activity was used as the control.

\section{Statistical analysis}

Data are expressed as mean \pm standard deviation (SD). Differences among experimental groups were analyzed by Students' $t$-tests or one-way analysis of variance followed by a post hoc Tukey's test using the GraphPad Prism 5.0 software (GraphPad, San Diego, CA, USA). A value less than 0.05 was considered significant.
A

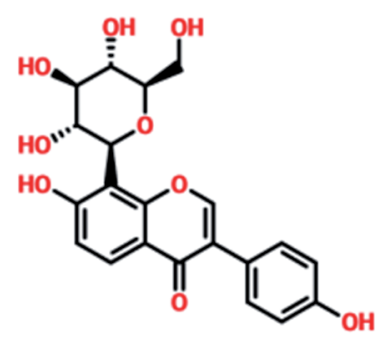

B

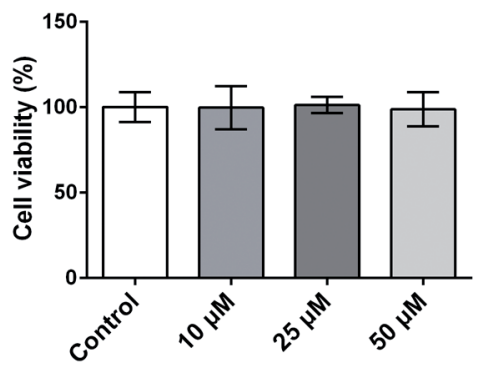

C

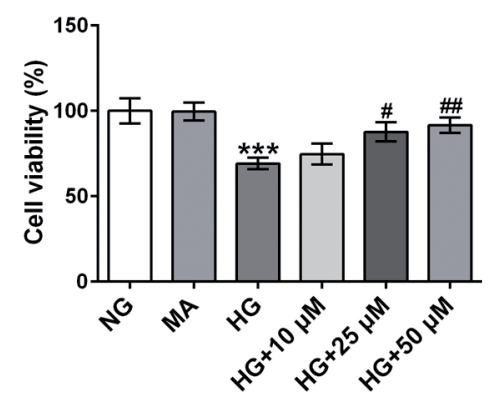

Figure 1. Puerarin treatment alleviated HG-induced inhibition of HRECs viability. A. The chemical structural formula of puerarin. B. Cell viability of HRECs after treatment with different concentrations of puerarin was examined using a CCK-8 assay kit. C. CCK- 8 assay kit was employed to evaluate cell viability after HRECs being treated with puerarin in HG condition. All experiments were performed in triplicate. Data are expressed as mean $\pm \mathrm{SD}$. ${ }^{* *} p<0.001 v$ s. MA group; ${ }^{\#} p<0.05,{ }^{\# \#} p<0.01 v$ s. HG group. NG, normal glucose; MA, mannitol; HG, high glucose; HRECs, human retinal endothelial cells. 


\section{Results}

\section{Puerarin treatment alleviated HG-induced inhibition of HRECs viability}

The chemical structural formula of puerarin is presented in Figure 1A. To study the effects of puerarin on HRECs stimulated by HG, different concentrations of puerarin were employed to treat HRECs, the viability of which was detected using a CCK-8 kit. As what is observed from Figure 1B, there were no relatively significant effects on cell viability in response to puerarin with 10, 20 and $50 \mu \mathrm{M}$, compared to the control group. By contrast with the MA group, HG challenge significantly inhibited cell viability, which was dose-dependently enhanced after puerarin treatment (Fig. 1C). $50 \mu \mathrm{M}$ of puerarin was selected to perform the following experiments. These results revealed that puerarin treatment increased cell viability in HG-induced HRECs.

\section{Puerarin treatment ameliorated HG-induced oxidative stress}

To investigate the effects of puerarin on oxidative stress induced by HG induction, the production of ROS and MDA were determined using corresponding kits. As displayed in Figure 2A and B, HG stimulation led to a notably increase in the contents of ROS and MDA in comparison to the MA group, whereas a significant decrease was observed by following puerarin treatment. Meanwhile, the activities of both SOD and CAT were dramatically reduced in the HG exposed group, which was remarkably enhanced after puerarin intervention (Fig. 2C,D). These observations implicated that puerarin treatment alleviated HG-induced oxidative stress.
Puerarin treatment attenuated HG-induced activation of pyroptosis

Subsequently, the effects of puerarin treatment on pyroptosis of HRECs were explored. Results shown in Figure 3 revealed that there was no obvious difference in cell pyroptosis between MA and NG groups. Conversely, HG stimulation notably elevated the level of pyroptosis in contrast to the NG group. When the HRECs were treated with both HG and puerarin, above change was partly abolished. Consistently, it was noticed that the expression of pyroptosis-related proteins including CASP1 and gasdermin-N domain (GSDMD-N) was markedly downregulated in the puerarin-treated group compared with the HG group (Fig. 4). These data had suggested that puerarin intervention relieved HG-induced activation of pyroptosis.

miR-16-5p inhibitor reversed the effects of puerarin on oxidative stress and pyroptosis in HG-stimulated HRECs

The expression of miR-16-5p was detected using RT-qPCR. As exhibited in Figure 5A, miR-16-5p level was dramatically reduced in the HG group in contrast to the MA group. However, compared to the HG group, puerarin treatment notably enhanced miR-16-5p expression level. The miR-16$5 p$ was silenced by transfection with miR-16-5p inhibitor (Fig. 5B) to analysis the changes of cell viability, oxidative stress and pyroptosis. Results in Figure $5 \mathrm{C}$ indicated that miR-16-5p silencing decreased cell viability compared with the $\mathrm{HG}+$ Puerarin+inhibitor NC group. Additionally, the level of ROS was notably elevated whereas the activities of SOD and CAT significantly decreased in the miR-16-5p inhibitor group (Fig. 5D-F). What's more, remarkably upregulated expression of CASP1 and GSDMD-N was
A

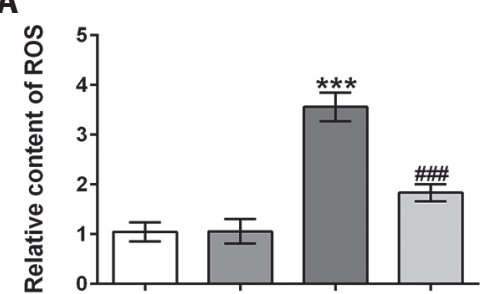

C

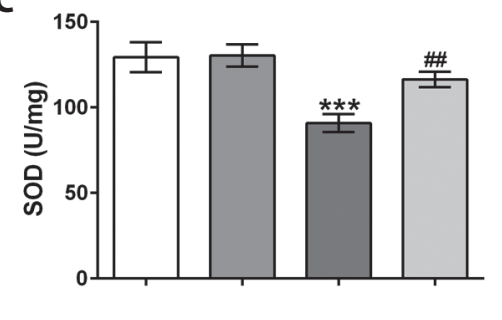

$\square$ NG
B

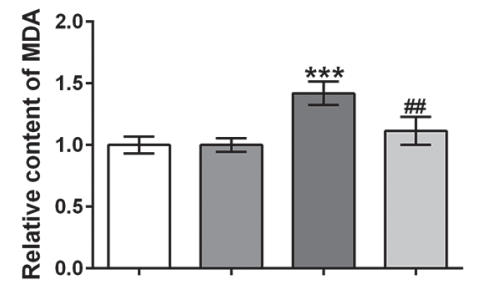

D

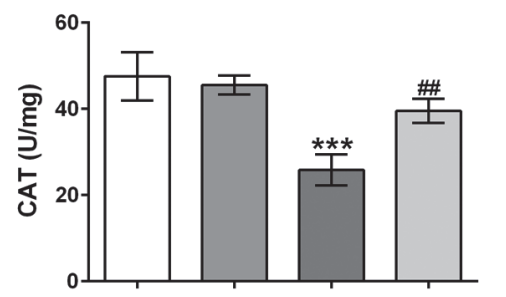

$\square$ HG+Puerarin
Figure 2. Puerarin treatment ameliorated HGinduced oxidative stress in HRECs. The contents of ROS (A) and MDA (B) were examined using corresponding kits. The activities of SOD (C) and CAT (D) were assessed using commercially available kits. All experiments were performed in triplicate. Data are expressed as mean \pm SD. ${ }^{* * *} p<0.001$ vs. MA group; ${ }^{\# \#} p<0.01,{ }^{\# \# \#} p<$ 0.001 vs. HG group. MA, mannitol; ROS, reactive oxygen species; MDA, malondialdehyde; SOD, superoxide dismutase; CAT, catalase. For more abbreviations, see Fig. 1. 

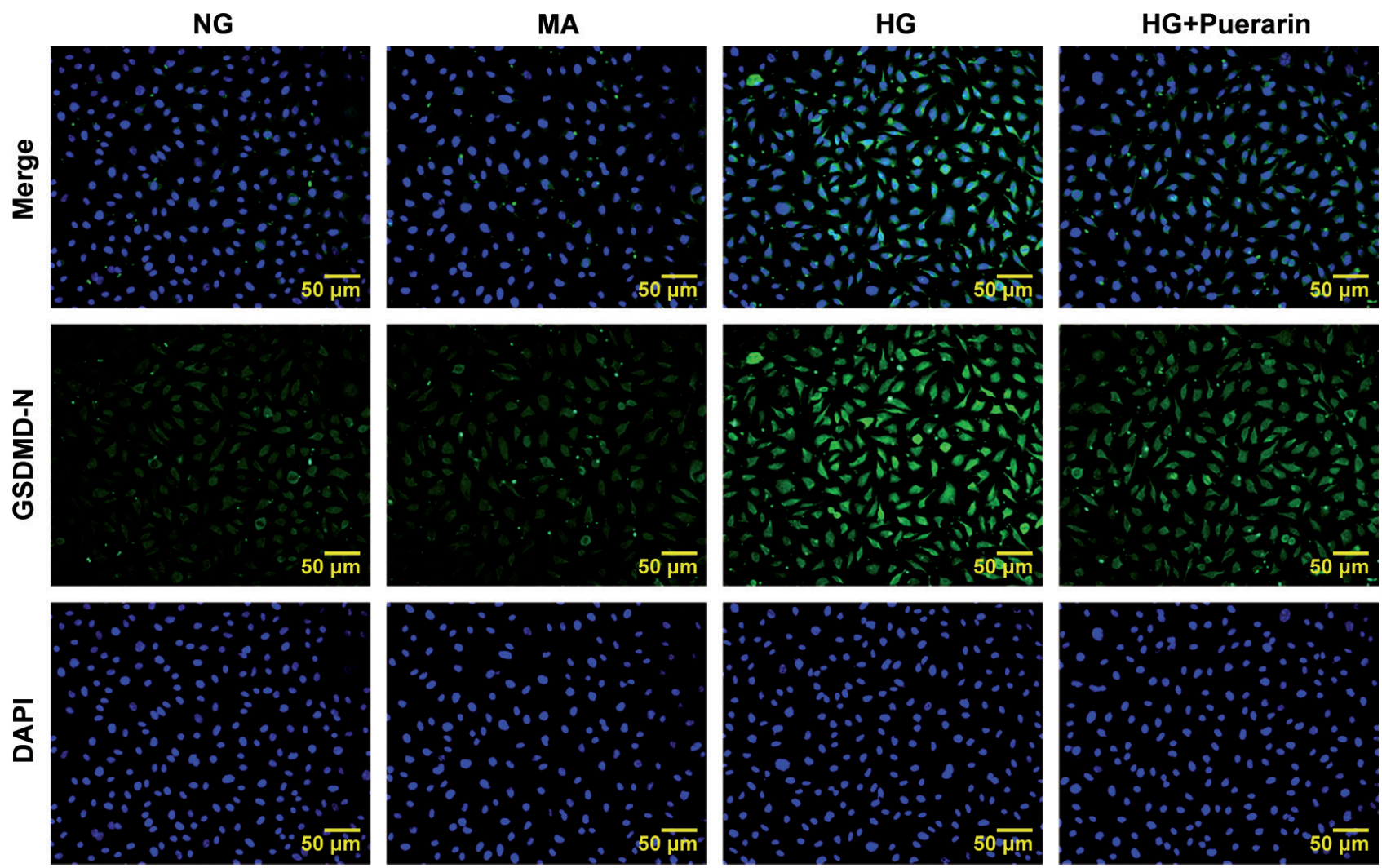

Figure 3. Puerarin treatment decreased the expression of GSDMA-N in HG-induced HRECs. Immunofluorescence assay was used to evaluate the level of GSDMA-N. Magnification, $\times 200$. All experiments were performed in triplicate. GSDMD-N, gasdermin-N domain. For more abbreviations, see Fig. 1.

observed when the HRECs treated with both HG and puerarin were transfected with miR-16-5p inhibitor (Fig. $5 \mathrm{G})$. Together, the data of present studies provide evidence that puerarin enhances miR-16-5p expression and $\mathrm{miR}-16-$ $5 p$ inhibitor reverses the inhibitory effects of puerarin on oxidative stress and pyroptosis in HG-stimulated HRECs.
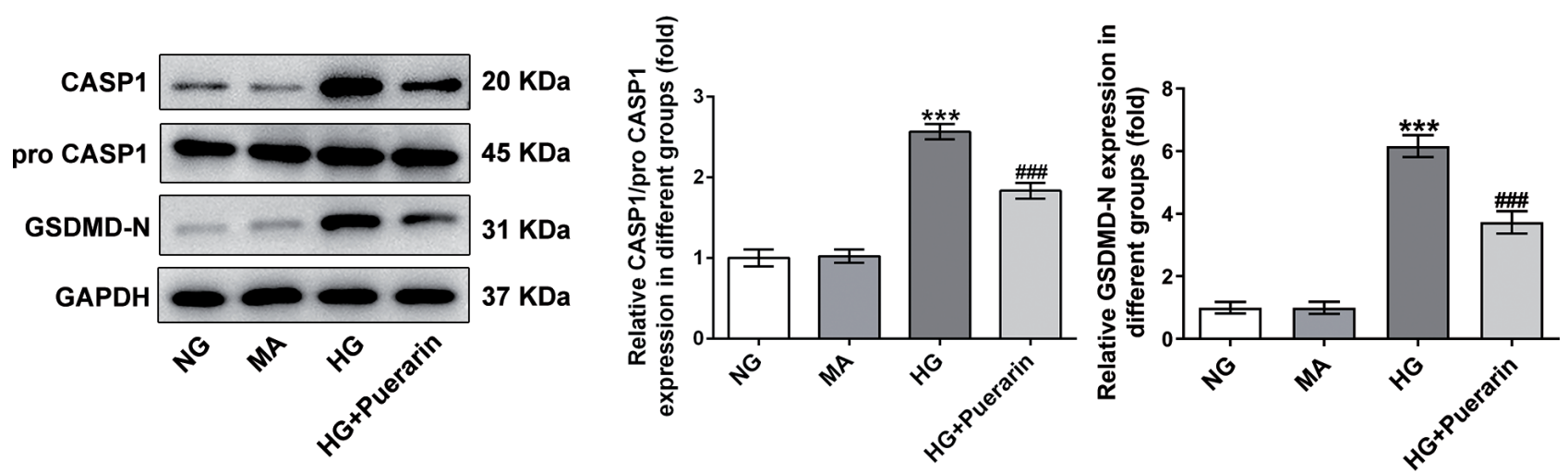

Figure 4. Puerarin treatment downregulated the expression of pyroptosis-related proteins in HG-induced HRECs. The expression of CASP1 and GSDMD-N was detected using Western blot analysis. All experiments were performed in triplicate. Data are expressed as mean \pm SD. ${ }^{* * *} p<0.001 v s$. MA group; ${ }^{\# \#} p<0.001 v s$. HG group. CASP1, caspase-1; GSDMD-N, gasdermin-N domain. For more abbreviations, see Fig. 1. 


\section{CASP1 was a direct target gene of miR-16-5p}

To further study the regulatory mechanisms of puerarin in HG-induced HRECs, the target gene of miR-16-5p was predicted by using Starbase database, and CASP 1 was found to be a potential target of miR-16-5p (Fig. 6A). After transfec- tion with miR-16-5p mimic (Fig. 6B), a luciferase reporter assay was employed to verify the target binding. Results in Figure 6C suggested that miR-16-5p mimic significantly decreased the luciferase activity in the CASP1 WT compared with the mimic NC group. Moreover, CASP1 expression was notably upregulated at the transcriptional level and
A

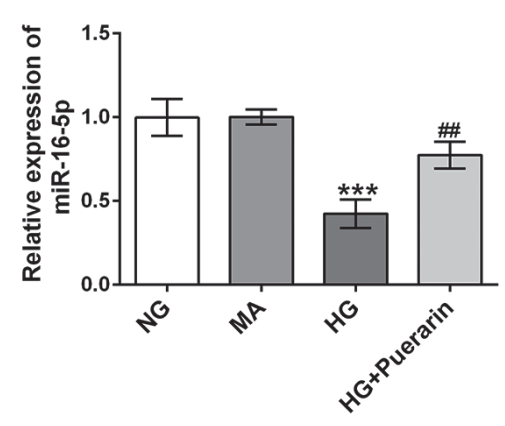

D

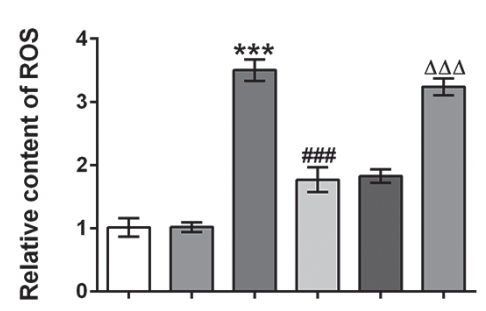

B

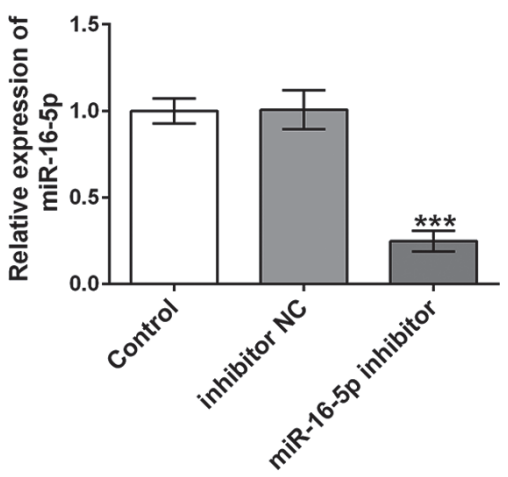

E

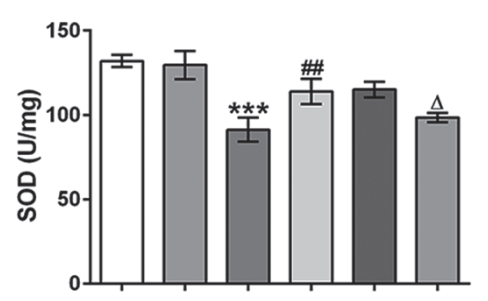

C

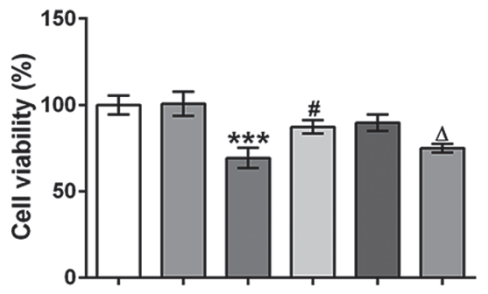

$\mathbf{G}$
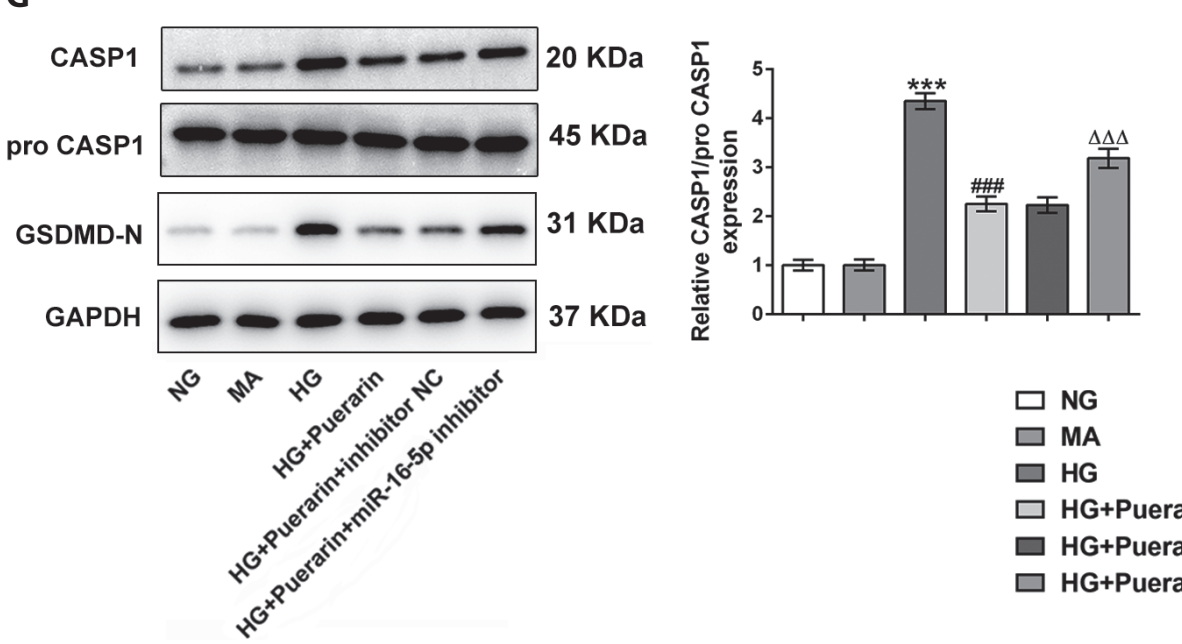

$\mathbf{F}$
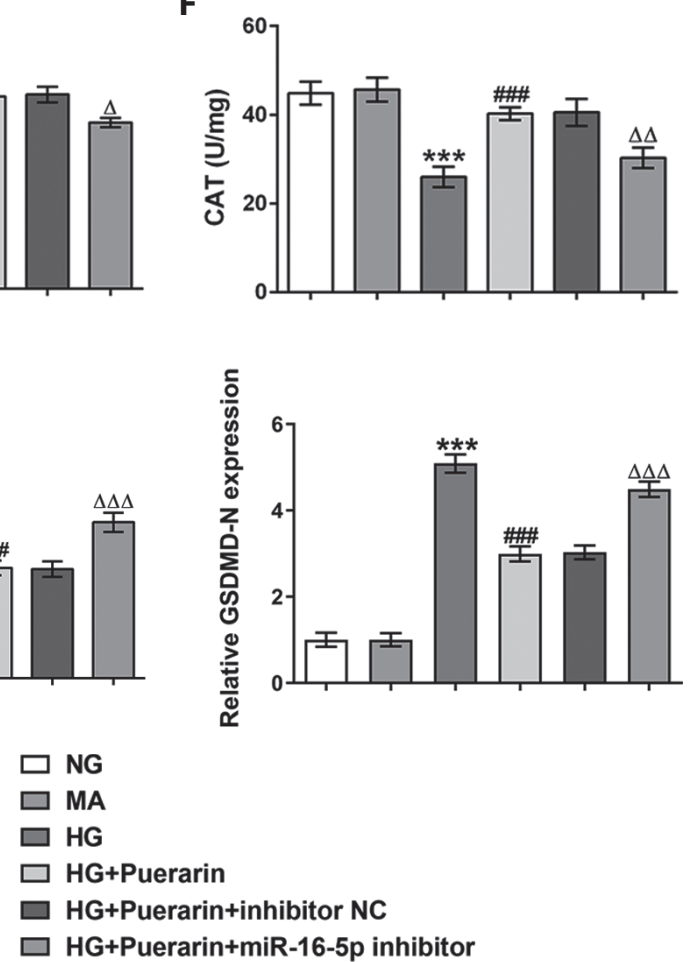

Figure 5. miR-16-5p inhibitor reversed the effects of puerarin on oxidative stress and pyroptosis in HG-stimulated HRECs. A. miR-16$5 p$ expression was measured using RT-qPCR. All experiments were performed in triplicate. Data are expressed as mean \pm SD. ${ }^{* * *} p<$ 0.001 vs. MA group; ${ }^{\# \#} p<0.01$ vs. HG group. B. miR-16-5p expression was detected by RT-qPCR. All experiments were performed in triplicate. Data are expressed as mean \pm SD. ${ }^{* *} p<0.001 v s$. inhibitor NC group. C. Cell viability was assessed using a CCK-8 assay kit. The contents of ROS (D) and the activities of SOD (E) CAT (F) were tested using corresponding kits. G. Western blot analysis was applied for determining the expression level of CASP1 and GSDMD-N. All experiments were performed in triplicate. Data are expressed as mean \pm SD. ${ }^{* * *} p<0.001$ vs. MA group; ${ }^{\#} p<0.05,{ }^{\# \#} p<0.01,{ }^{\# \# \#} p<0.001$ vs. HG group; ${ }^{\Delta} p<0.05,{ }^{\Delta \Delta} p<0.01,{ }^{\Delta \Delta \Delta} p<0.001 v s$. HG+Puerarin+inhibitor NC group. NC, negative control; ROS, reactive oxygen; SOD, superoxide dismutase; CAT, catalase; CASP1, caspase-1; GSDMD-N, gasdermin-N domain. For more abbreviations, see Fig. 1. 
A

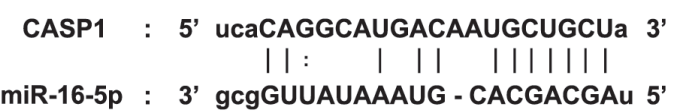

B

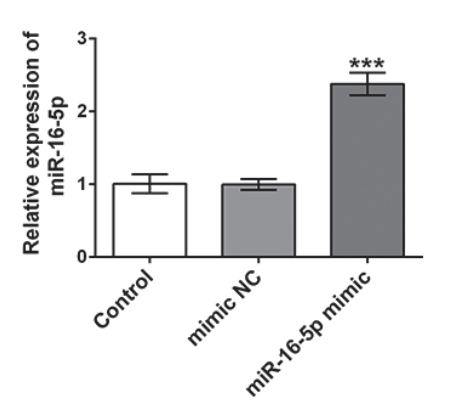

C

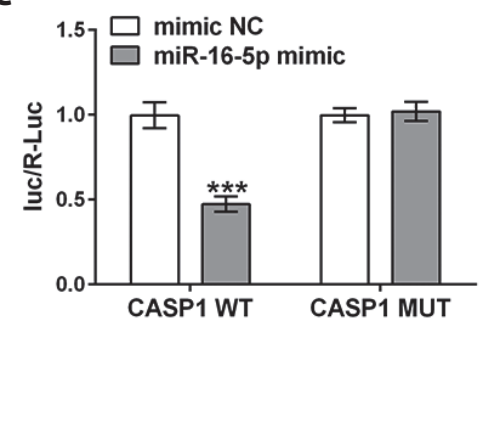

D

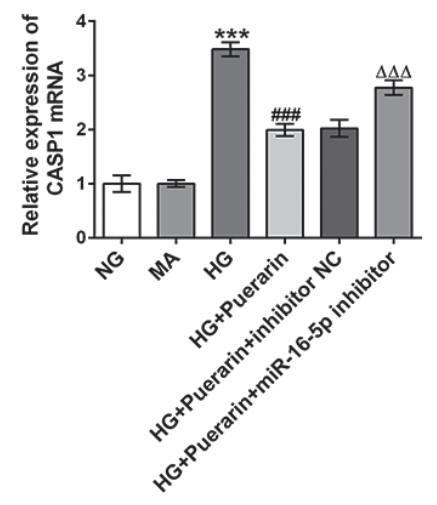

$\mathbf{E}$

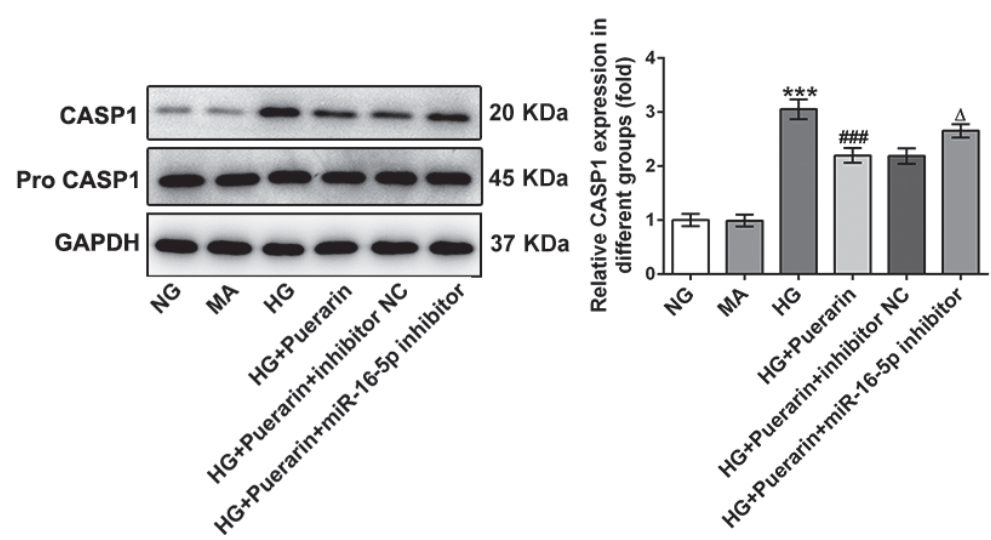

Figure 6. CASP1 was a direct target gene of miR-16-5p. A. Predicted target binding region between miR-16-5p and CASP1. B. RTqPCR was used to evaluate the expression of miR-16-5p after transfection with miR-16-5p mimic. All experiments were performed in triplicate. Data are expressed as mean $\pm \mathrm{SD}$. ${ }^{\star * \star} p<0.001 v s$. mimic NC group. C. Relative luciferase activities were detected using a luciferase reporter assay. All experiments were performed in triplicate. Data are expressed as mean \pm SD. ${ }^{* * *} p<0.001 v s$. mimic NC group. CASP1 mRNA and protein expression were determined using RT-qPCR (D) and Western blot analysis (E), respectively. All experiments were performed in triplicate. Data are expressed as mean \pm SD. ${ }^{* * *} p<0.001 v$ s. MA group; ${ }^{\# \#} p<0.001 v$ s. HG group; ${ }^{\Delta} p<$ $0.05,{ }^{\Delta \Delta \Delta} p<0.001 v s$. HG+Puerarin+inhibitor NC group. NC, negative control; CASP1, caspase-1; WT, Wild-type; MUT, mutant. For more abbreviations, see Fig. 1 .

post-transcriptional level in the $\mathrm{HG}+$ Puerarin+miR-16-5p inhibitor group by contrast with the $\mathrm{HG}+$ Puerarin+inhibitor NC group (Fig. 6D,E). To be brief, these data suggested that CASP1 was a direct target gene of miR-16-5p.

\section{Discussion}

DR is a prolonged hurdle occurring in patients with diabetes due to persistent high blood glucose level, which might lead to vision loss if untreated (Elsherbiny et al. 2013). A growing body of literature has shown that hyperglycemia results in a rise in ROS production, which is considered as a major predisposing factor in the development of DR (Calderon et al. 2017; Li et al. 2017). The excessive accumulation of ROS triggers the imbalance of oxidation and antioxidant, namely oxidative stress. Previous clinical experiments have clearly confirmed that oxidative stress plays a dominant role in the pathogenesis of DR (Wu et al. 2014; Kowluru et al. 2015). Therefore, antioxidant agents are deemed to be the underlying treatment of DR. MDA, the end product of lipid peroxidation, is an oxidative stress marker. Occurrence of oxidative stress enhanced the content of MDA while reducing the activities of antioxidant enzymes including SOD and CAT, which are crucial antioxidant enzymes in the defensive system under oxidative stress condition and they together resist the HRECs to HG-induced cell death (Xiao et al. 2020). The inhibitory effects of puerarin on oxidative stress have been reported in a large number of studies (Wu et al. 2014; Xue et al. 2016; Wang et al. 2017; Yue et al. 2018). It has been reported that puerarin inhibits HG-induced MCP-1 expression in cultured endothelial cells (Han et al. 2015). Importantly, Lian et al. (2019) demonstrated that puerarin suppresses HG-induced endothelial dysfunction through inhibiting endothelial Nlrp3 
inflammasome activation via ROS-dependent oxidative pathway. Chen et al. (2012) found that puerarin is able to enhance SOD activity in retinas of streptozotocin-induced early diabetic rats. The present study demonstrated that puerarin alleviates HG-induced oxidative stress in HRECs, suggesting a potential of puerarin being applied to the treatment of DR.

Pyroptosis is identified as inflammatory caspases (mainly CASP1)-dependent programmed cell death (Bergsbaken et al. 2009). Accumulating evidence shows that oxidative stressinduced pyroptosis is closely related to the development of multiple diseases (Liu et al. 2020; Zhou et al. 2020). What's more, a recent study found that pyroptosis is involved in the progression of $\mathrm{DR}$, which can be mediated by oxidative stress (Xi et al. 2020). It is worth noticing that puerarin can inhibit CASP1 activation by reducing ROS generation (Lian et al. 2019). GSDMD-N, a GSDMD protein cleaved by caspases to generate a gasdermin- $\mathrm{N}$ domain, moves to the plasma membrane to exhibit pore-forming activity (Jorgensen and Miao 2015; Kuang et al. 2017). Therefore, GSDMD-N acts as the final and direct executor of pyroptosis. The current study for the first time revealed that puerarin markedly decreases the expression of GSDMD-N and CASP1, providing evidence of the inhibitory effects of puerarin on pyroptosis in HG-stimulated HRECs.

To further investigate the underlying regulatory mechanisms of puerarin in HG-induced HRECs, the expression of miR-16-5p was determined after puerarin treatment. And significantly upregulated miR-16-5p expression following puerarin exposure in HG condition was observed in this study. Moreover, miR-16 was reported to suppress insulin resistance so as to protect HRECs from hyperglycemia-induced apoptosis (Ye and Steinle 2015). A recent study has proven that miR-16 could alleviate retinal leukostasis through decreased pro-inflammatory signaling in HRECs (Ye et al. 2016). Numerous studies have indicated that miRNAs can modulate gene expression at the posttranscriptional level via inducing direct mRNA degradation or translational repression, which ultimately affects multiple cellular processes included development, growth and metastasis (Lin and Gregory 2015; Weng et al. 2016; Vafadar et al. 2019). The Starbase database predicts that CASP1, a major mediated factor of pyroptosis is a potential target of miR-16-5p, which was verified by the results of luciferase reporter assay. As expected, miR-16-5p inhibitor remarkably elevated CASP1 expression at the transcriptional level and post-transcriptional level compared to the inhibitor NC group. Based on these data, puerarin may inhibit oxidative stress and pyroptosis 232 by regulating miR-16-5p/CASP 1 axis in HG-stimulated HRECs.

\section{Conclusion}

In conclusion, the present study demonstrates that puerarin effectively ameliorates HG-induced oxidative stress and pyroptosis by targeted modulation of miR-16-5p/CASP1 axis, which is a new protection mechanism of puerarin on DR. These findings identify that puerarin may be one of the avenues towards the clinical treatment of DR.

Acknowledgements. This work was supported by the National Natural Science Foundation of China (81774419).

Conflict of interest. All authors declare that he/she has no conflict of interest.

\section{References}

Abu El-Asrar AM, Midena E, Al-Shabrawey M, Mohammad G (2013): New developments in the pathophysiology and management of diabetic retinopathy. J. Diabetes. Res. 2013, 424258 https://doi.org/10.1155/2013/424258

Bergsbaken T, Fink SL, Cookson BT (2009): Pyroptosis: host cell death and inflammation. Nat. Rev. Microbiol. 7, 99-109 https://doi.org/10.1038/nrmicro2070

Cai, Y, Zhang X, Xu X, Yu Y (2017): Effects of puerarin on the retina and STAT3 expression in diabetic rats. Exp. Ther. Med. 14, 5480-5484 https://doi.org/10.3892/etm.2017.5203

Calderon GD, Juarez OH, Hernandez GE, Punzo SM, De la Cruz ZD (2017): Oxidative stress and diabetic retinopathy: development and treatment. Eye (Lond) 31, 1122-1130 https://doi.org/10.1038/eye.2017.64

Chen F, Zhang HQ, Zhu J, Liu KY, Cheng H, Li GL, Xu S, Lv WH, Xie ZG (2012): Puerarin enhances superoxide dismutase activity and inhibits RAGE and VEGF expression in retinas of STZ-induced early diabetic rats. Asian. Pac. J. Trop. Med. 5, 891-896 https://doi.org/10.1016/S1995-7645(12)60166-7

Elsherbiny NM, Ahmad S, Naime M, Elsherbini AM, Fulzele S, Al-Gayyar MM, Eissa LA, El-Shishtawy M M, Liou GI (2013): ABT-702, an adenosine kinase inhibitor, attenuates inflammation in diabetic retinopathy. Life. Sci. 93, 78-88 https://doi.org/10.1016/j.lfs.2013.05.024

Han P, Gao DH, Zhang W, Liu SH, Yang SY, Li XJ (2015): Puerarin suppresses high glucose-induced MCP-1 expression via modulating histone methylation in cultured endothelial cells. Life Sci. 130, 103-107 https://doi.org/10.1016/j.lfs.2015.02.022

Jeon YD, Lee JH, Lee YM, Kim DK (2020): Puerarin inhibits inflammation and oxidative stress in dextran sulfate sodium-induced colitis mice model. Biomed. Pharmacother. 124, 109847 https://doi.org/10.1016/j.biopha.2020.109847

Jorgensen I, Miao EA (2015): Pyroptotic cell death defends against intracellular pathogens. Immunol. Rev. 265, 130-142 https://doi.org/10.1111/imr.12287

Kowluru RA, Kowluru A, Mishra M, Kumar B (2015): Oxidative stress and epigenetic modifications in the pathogenesis of diabetic retinopathy. Prog. Retin. Eye Res. 48, 40-61 https://doi.org/10.1016/j.preteyeres.2015.05.001

Kuang S, Zheng J, Yang H, Li S, Duan S, Shen Y, Ji C, Gan J, Xu XW, Li J (2017): Structure insight of GSDMD reveals the basis 
of GSDMD autoinhibition in cell pyroptosis. Proc. Natl. Acad. Sci. USA 114, 10642-10647

https://doi.org/10.1073/pnas.1708194114

Li C, Miao X, Li F, Wang S, Liu Q, Wang Y, Sun J (2017): Oxidative stress-related mechanisms and antioxidant therapy in diabetic retinopathy. Oxid. Med. Cell. Longev. 2017, 9702820 https://doi.org/10.1155/2017/9702820

Lian DW, Yuan HQ, Yin XZ, Wu YJ, He RR, Huang Y, Chen Y (2019): Puerarin inhibits hyperglycemia-induced interendothelial junction through suppressing endothelial Nlrp3 inflammasome activation via ROS-dependent oxidative pathway. Phytomedicine 55, 310-319

https://doi.org/10.1016/j.phymed.2018.10.013

Lin S, Gregory RI (2015): MicroRNA biogenesis pathways in cancer. Nat. Rev. Cancer 15, 321-333

https://doi.org/10.1038/nrc3932

Liu J, Bhuvanagiri S, Qu XH (2019): The protective effects of lycopus lucidus turcz in diabetic retinopathy and its possible mechanisms. Artif. Cells. Nanomed. Biotechnol. 47, 2900-2908 https://doi.org/10.1080/21691401.2019.1640230

Liu SS, Du J, Li DF, Yang PP, Kou YY, Li CS, Zhou Q, Lu YP, Hasegawa T, Li MQ (2020): Oxidative stress induced pyroptosis leads to osteogenic dysfunction of MG63 cells. J. Mol. Histol. 51, 221-232 https://doi.org/10.1007/s10735-020-09874-9

Liu X, Li S, Li Y, Cheng B, Tan B, Wang G (2018): Puerarin inhibits proliferation and induces apoptosis by upregulation of miR-16 in bladder cancer cell line T24. Oncol. Res. 26, 1227-1234 https://doi.org/10.3727/096504018X15178736525106

Livak KJ, Schmittgen TD (2001): Analysis of relative gene expression data using real-time quantitative PCR and the 2(-Delta Delta C(T)) Method. Methods 25, 402-408 https://doi.org/10.1006/meth.2001.1262

Mohamed R, Sharma I, Ibrahim AS, Saleh H, Elsherbiny NM, Fulzele S, Elmasry K, Smith SB, Al-Shabrawey M, Tawfik A (2017):. Sci. Rep. 7, 11 - chyba nazov clanku https://doi.org/10.1038/s41598-017-09731-y

Ramanathan K, Padmanabhan G (2020): MiRNAs as potential biomarker of kidney diseases: A review. Cell Biochem. Funct. 38, 990-1005 https://doi.org/10.1002/cbf.3555

Vafadar A, Shabaninejad Z, Movahedpour A, Mohammadi S, Fathullahzadeh S, Mirzaei HR, Namdar A, Savardashtaki A, Mirzaei H (2019): Long non-coding RNAs as epigenetic regulators in cancer. Curr. Pharm. Des. 25, 3563-3577 https://doi.org/10.2174/1381612825666190830161528

Wang CJ, Yao JH, Ju LJ, Wen XH, Shu L (2020): Puerarin ameliorates hyperglycemia in HFD diabetic mice by promoting beta-cell neogenesis via GLP-1R signaling activation. Phytomedicine 70, 153222 https://doi.org/10.1016/j.phymed.2020.153222

Wang K, Zhu X, Zhang K, Yao Y, Zhuang M, Tan CY, Zhou FF, Zhu L (2017): Puerarin inhibits amyloid beta-induced NLRP3 inflammasome activation in retinal pigment epithelial cells via suppressing ROS-dependent oxidative and endoplasmic reticulum stresses. Exp. Cell Res. 357, 335-340 https://doi.org/10.1016/j.yexcr.2017.05.030

Weng JH, Yu CC, Lee YC, Lin CW, Chang WW, Kuo YL (2016): miR-494-3p induces cellular senescence and enhances radio- sensitivity in human oral squamous carcinoma cells. Int. J. Mol. Sci. 17, 1092 https://doi.org/10.3390/ijms17071092

Wu Y, Yu CC, Lee YC, Lin CW, Chang WW, Kuo YL (2014): Oxidative stress: implications for the development of diabetic retinopathy and antioxidant therapeutic perspectives. Oxid. Med. Cell. Longev. 2014, 752387 https://doi.org/10.1155/2014/752387

Xi XT, Yang YN, Ma J, Chen QB, Zeng Y, Li JX, Chen L, Li Y (2020): MiR-130a alleviated high-glucose induced retinal pigment epithelium (RPE) death by modulating TNF-alpha/SOD1/ ROS cascade mediated pyroptosis. Biomed. Pharmacother. 125, 109924 https://doi.org/10.1016/j.biopha.2020.109924

Xiao H, Yang H, Zeng Y (2020): Long non-coding RNA Arid2-IR affects advanced glycation end products-induced human retinal endothelial cell injury by binding to Smad3. Int. Ophthalmol. 40, 1123-1133 https://doi.org/10.1007/s10792-019-01277-4

Zhu X, Wang K, Zhang K, Lin XF, Zhu L, Zhou FF (2016): Puerarin protects human neuroblastoma SH-SY5Y cells against glutamate-induced oxidative stress and mitochondrial dysfunction. J. Biochem. Mol. Toxicol. 30, 22-28 https://doi.org/10.1002/jbt.21736

Yang Q, Li S, Zhou Z, Fu M, Yang X, Hao K, Liu Y (2020): HDAC6 inhibitor Cay10603 inhibits high glucose-induced oxidative stress, inflammation and apoptosis in retinal pigment epithelial cells via regulating NF-kappaB and NLRP3 inflammasome pathway. Gen. Physiol. Biophys. 39, 169-177 https://doi.org/10.4149/gpb_2019058

Ye EA, Liu L, Jiang Y, Jan J, Gaddipati S, Suvas S, Steinle JJ (2016): $\mathrm{miR}-15 \mathrm{a} / 16$ reduces retinal leukostasis through decreased proinflammatory signaling. J. Neuroinflammation 13, 305 https://doi.org/10.1186/s12974-016-0771-8

Ye EA, Steinle JJ (2015): miR-15b/16 protects primary human retinal microvascular endothelial cells against hyperglycemiainduced increases in tumor necrosis factor alpha and suppressor of cytokine signaling 3. J. Neuroinflammation 12, 44 https://doi.org/10.1186/s12974-015-0265-0

Yue J, Chang SW, Xiao ZX, Qi YF, He JX (2018): The protective effect of puerarin on angiotensin II-induced aortic aneurysm formation by the inhibition of NADPH oxidase activation and oxidative stress-triggered AP-1 signaling pathways. Oncol. Lett. 16, 3327-3332 https://doi.org/10.3892/ol.2018.9021

Zhang TH, Huang CM, Gao X, Wang JW, Hao LL, Ji Q (2018): Gastrodin inhibits high glucoseinduced human retinal endothelial cell apoptosis by regulating the SIRT1/TLR4/NFkappaBp65 signaling pathway. Mol. Med. Rep. 17, 7774-7780 https://doi.org/10.3892/mmr.2018.8841

Zhou Y, Li KS, Liu L, Li SL (2020): MicroRNA-132 promotes oxidative stress-induced pyroptosis by targeting sirtuin 1 in myocardial ischaemia-reperfusion injury. Int. J. Mol. Med. 45, 1942-1950 https://doi.org/10.3892/ijmm.2020.4557

Received: December 21, 2020

Final version accepted: March 23, 2021 